\title{
Enhancement of Photodetector Responsivity in Standard SOI CMOS Processes by introducing Resonant Grating Structures
}

M. Auer

max.auer@ziti.uni-heidelberg.de

K.-H. Brenner
University of Heidelberg, 68131 Mannheim, Germany

University of Heidelberg, 68131 Mannheim, Germany

A new photodetector concept is described, which is fully compatible with the standard SOI CMOS process and does not require any postprocessing steps. Our simulations are based on two-dimensional RCWA (Rigorous Coupled Wave Analysis) and local absorption theory (K.-H. Brenner, "Aspects for calculating local absorption with the rigorous coupled-wave method" Optics Express 2010, Vol. 18, Iss. 10, pp. 10369-10376, (2010)). The simulations show that optimized lateral grating structures are able to enhance the absorption efficiency of thin semi-conductor detectors by a factor of 32 compared to non-enhanced approaches. [D0I: 10.2971/je0s.2011.11014S]

Keywords: local absorption, RCWA, photodetector design

\section{INTRODUCTION}

In order to optimise the design of photodetectors, photovoltaic elements or photolithographic setups, it is a common procedure to simulate intensity distributions. Therefore tools based on various theories are used, like scalar diffraction theories and rigorous methods in the time or modal domain. While all these theories provide sufficiently exact results for the intensity, they do not express the relevant quantity for describing detector performance. For detector responsivity, the important quantity is the absorbed power in a given volume element. A common way to describe absorption is based on the Lambert Beer law, which only applies to homogeneous volumes.

Based on RCWA (Rigorous Coupled Wave Analysis), which provides the electromagnetic field distribution for structured volumes, a method to calculate local absorption was presented in [1]. In the following, this method is called the local absorption theory. Local absorption has also been calculated before [2] by taking the divergence of the Poynting vector.

As an application example, the present paper demonstrates a new concept of a photodetector design [3], which is based on simulation results with the local absorption theory. This photodetector is designed for a wavelength of $850 \mathrm{~nm}$ and is fully compatible with the standard SOI CMOS process eliminating the need for any post-processing steps. Using optimised lateral grating structures, it was accomplished to exceed global absorption values of $68 \%$ in Silicon layers of only $100 \mathrm{~nm}$ thickness, while the non-enhanced absorption only reaches $2 \%$. Unlike resonant-cavity-enhanced photonic devices [4]-[6] where light is reflected like in a Fabry-Perot type resonator, our lateral grating structures have been optimised for a certain wavelength to minimise Fresnel reflections and form resonant structures. Thereby the absorption can be enhanced due to diffraction effects that can concentrate light in a desired re- gion. This mechanism alone results in a significant increase of responsivity for the detector. Being compatible with SOI CMOS implies that this detector design can use the same materials and manufacturing steps as the CMOS technology itself. Thus no further process development is needed. Moreover the fabrication process ends up with a very compact design.

\section{LOCAL ABSORPTION THEORY}

We assume that the electromagnetic field distribution of a considered volume is known from RCWA calculations or similar methods. Traditionally, the RCWA provides diffraction order efficiencies for reflection and transmission. A value for global absorption is only obtained by applying the principle of energy conservation. It is calculated by adding up all reflection and transmission efficiencies:

$$
\begin{aligned}
& D^{\circ O R_{m}}=\operatorname{Re}\left(\frac{k_{I, z m}}{k_{0} n_{I} \cos (\theta)}\right)\left|R_{m}\right|^{2} \\
& \mathrm{DOT}_{m}=\operatorname{Re}\left(\frac{k_{I I, z m}}{k_{0} n_{I} \cos (\theta)}\right)\left|T_{m}\right|^{2} \\
& D O A=1-\sum_{m} \mathrm{DOR}_{m}-\sum_{m} \mathrm{DOT}_{m}
\end{aligned}
$$

It was shown in [1] that the relative absorbed power can be expressed as:

$$
\frac{P_{a}}{P_{i}}=\frac{k_{0}^{2}}{k_{i, z}} \frac{1}{A} \cdot \iiint \operatorname{Im}(\varepsilon(\mathbf{r})) \cdot\left|\mathbf{E}_{\mathbf{1}}(\mathbf{r})\right|^{2} d V
$$

The formula describes the ratio of absorbed power to incident power for an illumination with a plane wave and a wave number $k_{0} . A$ is the illuminated area and the incident direction is expressed by the $\mathrm{z}$-component of the incident $\mathrm{k}$-Vektor $k_{i, z}$. 
The absorbed power in a volume element $d V$ is mainly determined by the imaginary part of epsilon and the relative field intensity. This quantity is the response to an incident illumination amplitude of 1 and is readily availably from almost all rigorous simulation tools.

After discretisation, the volume integral can be approximated by Riemann-sums:

$$
\frac{P_{a}}{P_{i}}=\frac{\delta z}{N X \cdot N Y} \frac{k_{0}^{2}}{k_{i, z}} \cdot \sum_{j, k, l} \operatorname{Im}\left(\varepsilon\left(\mathbf{r}_{j, k, l}\right)\right) \cdot\left|\mathbf{E}_{1}\left(\mathbf{r}_{j, k, l}\right)\right|^{2}
$$

In this form all terms of the equation are either input parameters or results from the RCWA and every single term under the Riemann-sum represents the local power absorbed in a volume element $j, k, l$ of size $\delta x_{j} \delta y_{k} \delta z_{l}$. The summation for $j$ extends from 0 to $N X-1$, for $k$ from 0 to $N Y-1$ and for $l$ from 0 to $N Z-1$. Further in this article we will refer to this summation result as the integrated absorption. Since the theory starts with the law of energy conservation and is exact, the integrated absorption theoretically should be equal to the gobal absorption (cf. Eq. (3)) of the RCWA. Expression (5) can easily be implemented in a RCWA algorithms and is the origin of all simulations in this paper.

\section{PHOTODETECTOR DESIGN}

Photodetectors based on semi-conductor technology are photodiodes or phototransistors. In any case, the measurable photocurrent depends on the amount of light absorbed in the depletion region. A simple analysis of Eq. (4) reveals that the requirements for high absorption values are a high imaginary epsilon, high intensity and a large volume. Unfortunately this is not what we normally get, if we try to use diodes fabricated by standard SOI CMOS technology as photodiodes. In CMOS technology, the ultra thin layer designs limit the height of the active zone and high doping levels cause narrow depletion regions (cf. Figure 1). Furthermore, the assortment of available materials is limited and for wavelengths above $850 \mathrm{~nm}$, Silicon is almost transparent.

In order to enhance absorption under these circumstances, our approach utilises one of the CMOS layers as a resonance grating, which reduces Fresnel reflections and concentrates the incident light inside the depletion regions. To stay conform with the CMOS process, the grating was realised by structuring the poly-silicon layer, which usually forms the transistor gates in the layer above the active zone.

Grating period $(p)$ and gate width $(w)$ form a two-dimensional parameter space (cf. Figure 2), which can be scanned for absorption maxima. Figure 3 shows a parameter scan of the global absorption values (DOA) at normal incidence of TEpolarised light. The underlaying algorithm is based on the RCWA according to Eq. (3). The parameter scan shows two strong absorption maxima $(\mathrm{G} 1, \mathrm{G} 2)$ with a peak absorption of $86.15 \%$ at $\mathrm{G} 1(p=0.275 \mu \mathrm{m}$ and $w=0.82 p)$.

However from this picture it cannot be determined how much light is absorbed in each layer. Thus it cannot be ruled out that

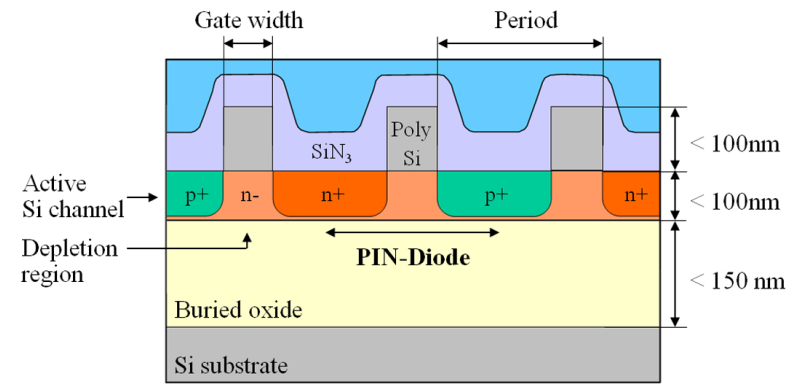

FIG. 1 Detector layout.

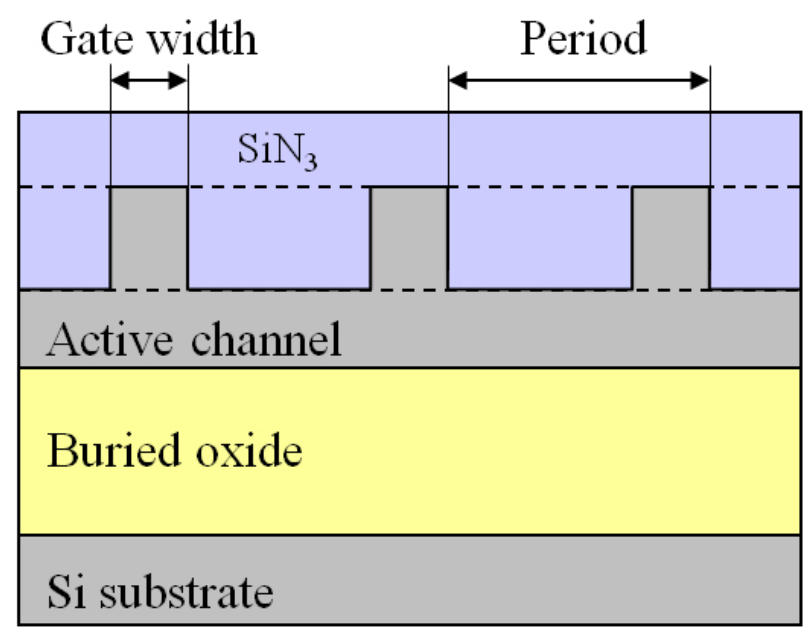

FIG. 2 Scanning parameters for the simulation: Grating period $p[\mu \mathrm{m}]$ and gate width $w[p]$ (The gate width is measured in multiples of the grating period).

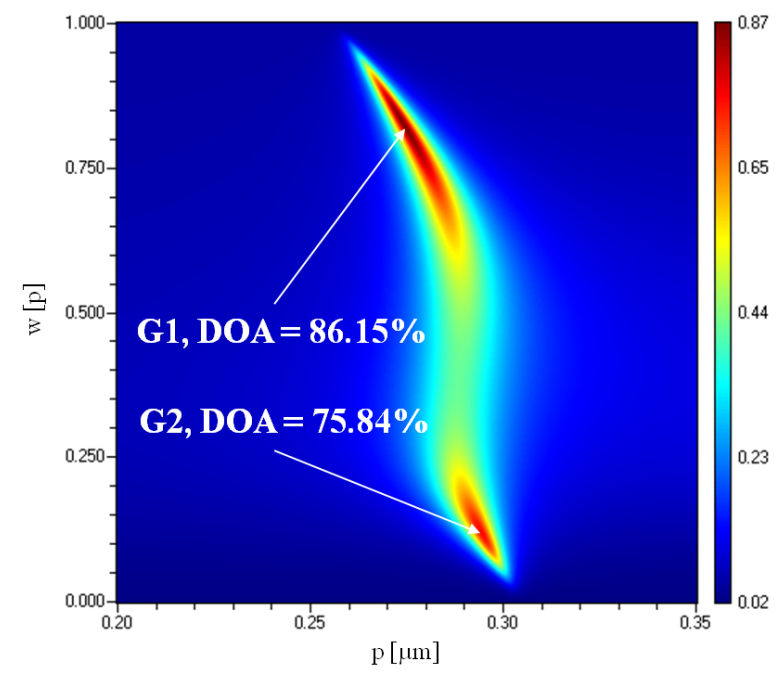

FIC. 3 Parameter scan of global absorption at normal incidence of TE-polarised light (wavelength $\lambda=850 \mathrm{~nm}$ ). The global absorption values are normalised with respect to the incident energy. All Simulations were done with 21 modes.

the maximum of global absorption and the absorption maximum of the active layer may not match. Figure 4 and 5 show exactly this phenomenon, which will be discussed in the following.

The same parameter scan is made for local absorption in the grating and in the active layer separately. It reveals that in the active layer, at parameter configuration G1 (=L1), the local absorption is only $59.6 \%$, whereas a different parameter config- 


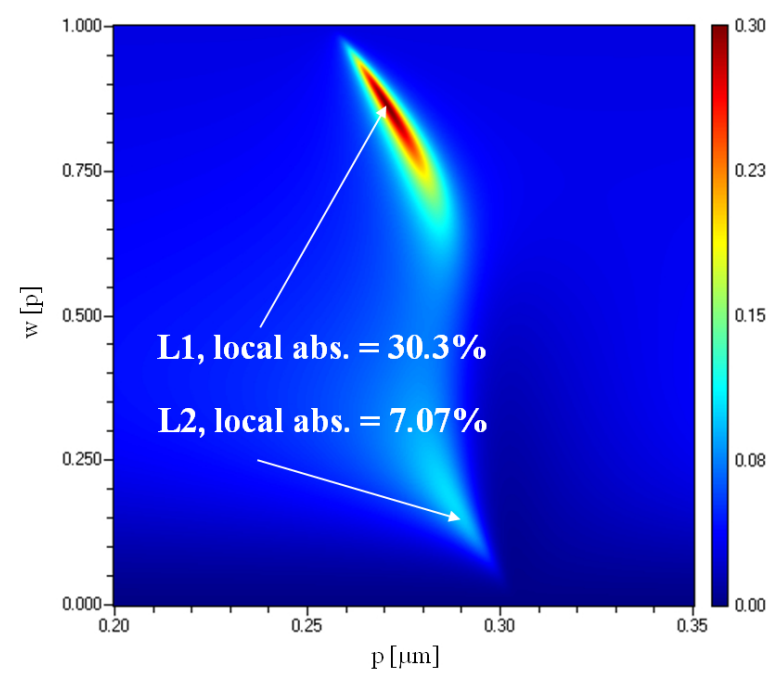

FIG. 4 Local absorption in the grating layer (TE-polarised light).

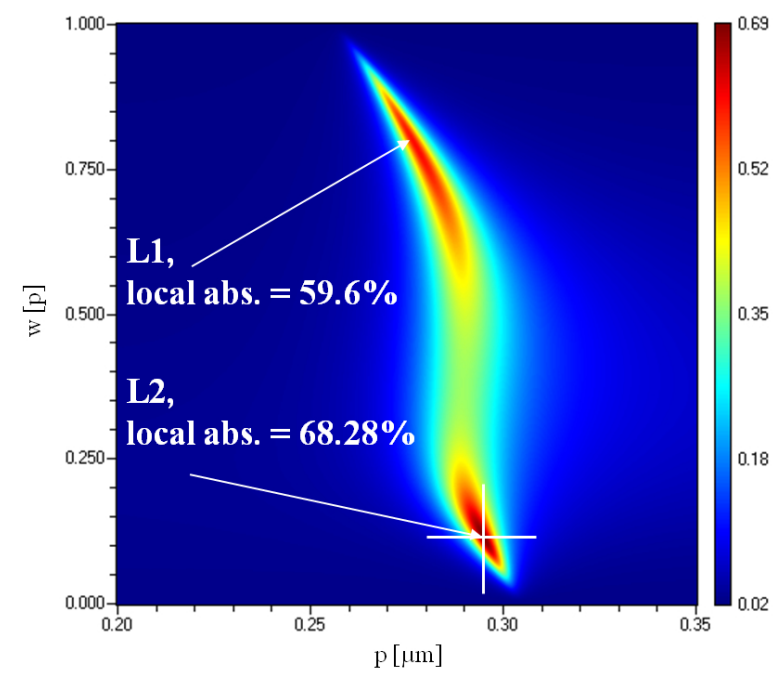

FIG. 5 Local absorption in the active channel layer (TE-polarised light).

uration (L2) reaches a local absorption maximum of $68.28 \%$ (cf. Figure 5). The selection of the peak absorption from the parameter space has optimised the intensity as well as the local absorption to be maximal in the active zone. Moreover, we were fortunate to achieve that the absorption maxima appear right under the gates in the depletion region. This should be beneficial for the generation of the photocurrent. Compared to the non-enhanced absorption, reaching only a value of $2.14 \%$, which can be taken e.g. from Figure 5 at $w=1$, the enhanced apsorption of $68.28 \%$ means an increase of efficiency by a factor of 32 !

In Figure 6 the detailed intensity distribution and the local absorption in a two-dimensional $(x, z)$-cross-sectional map of the grating is shown for L2. It visualises the effect of the grating optimisation as it concentrates intensity in the active zone and thereby increases the local absorption in the depletion region.

For fabrication, tolerances are important characteristics. As shown in Figure 7 the manufacturing requirements for the grating period are much higher than for the gate widths. This actually works in our favour, because the accuracy of the grating period is only dependent on the lithographic precision,
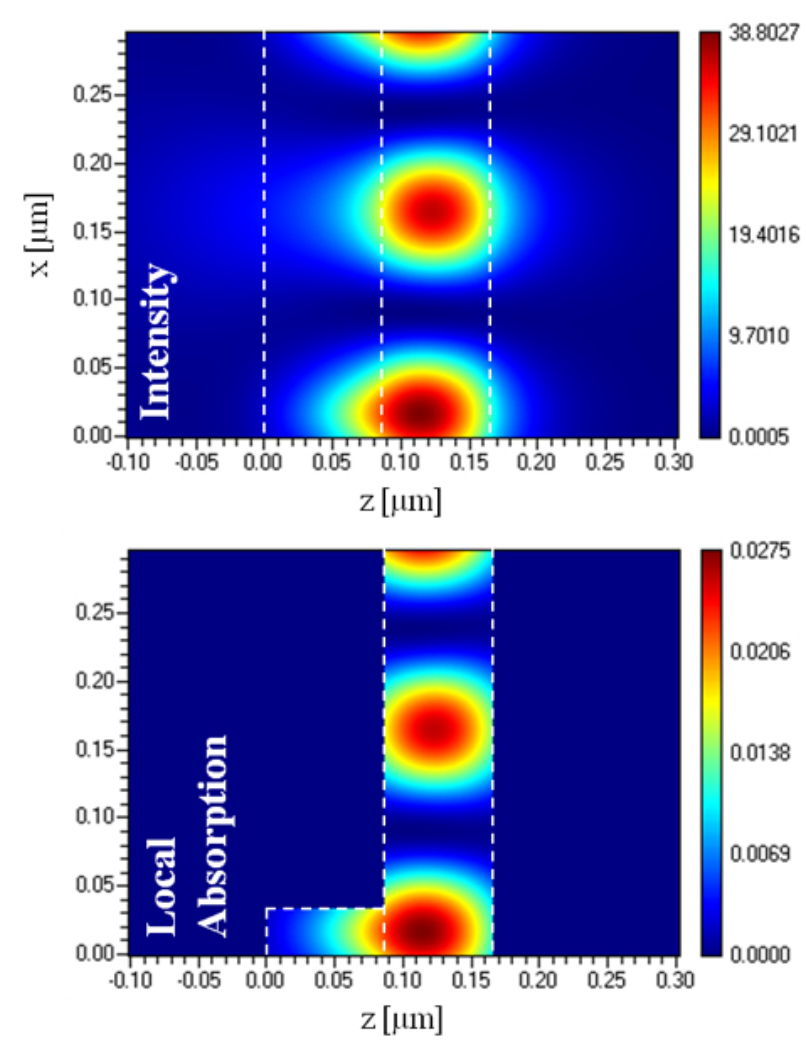

FIG. 6 Intensity and local absorption at $\mathrm{G}_{2}(p=0.295 \mu \mathrm{m}$ and $w=0.113 p)$ (TE-polarised light with normal angle of incidence).
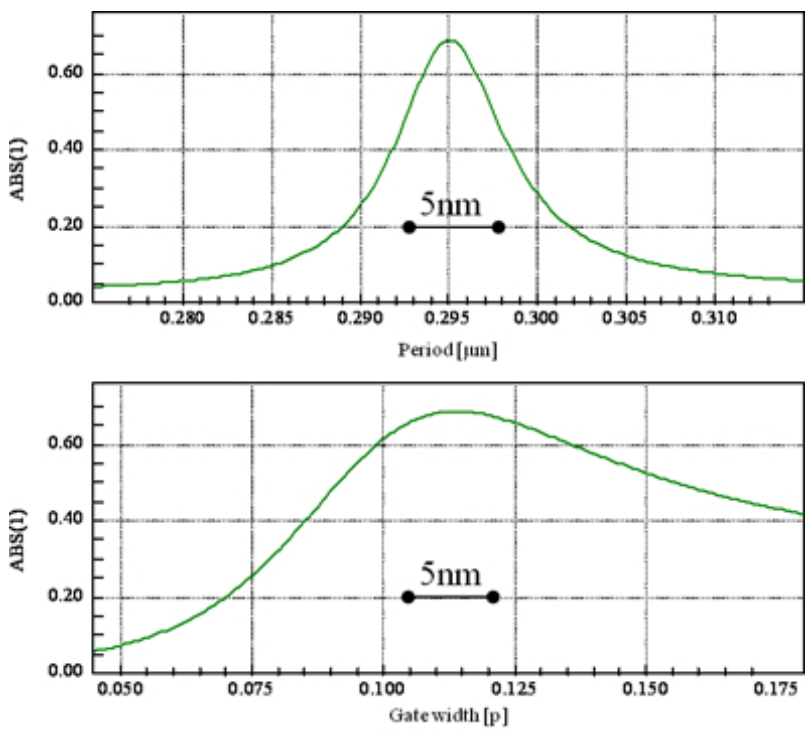

FIG. 7 Tolerance analysis of the local absorption in the active layer at $\mathrm{C}_{2}(p=0.295 \mu \mathrm{m}$ and $w=0.113 p$ ) by varying the period (top) and the gate width (bottom). Incident light: TE-polarised at normal incidence.

whereas the precision of the gate width depends on development chemistry, etch rates etc. and is thus less accurate.

Similar considerations apply to TM-polarisation, although in this case the absorption maxima G1 and G2 are shifted towards larger periods. At parameter configuration G1 the global absorption is just slightly lower than in the TE-case (cf. G1: 83.15\% in Figure 8 and G1: 86.15\% in Figure 3), but distributed equally among the two layers at L1. At parameter configuration $\mathrm{G} 2$ the resonantly enhanced absorption al- 


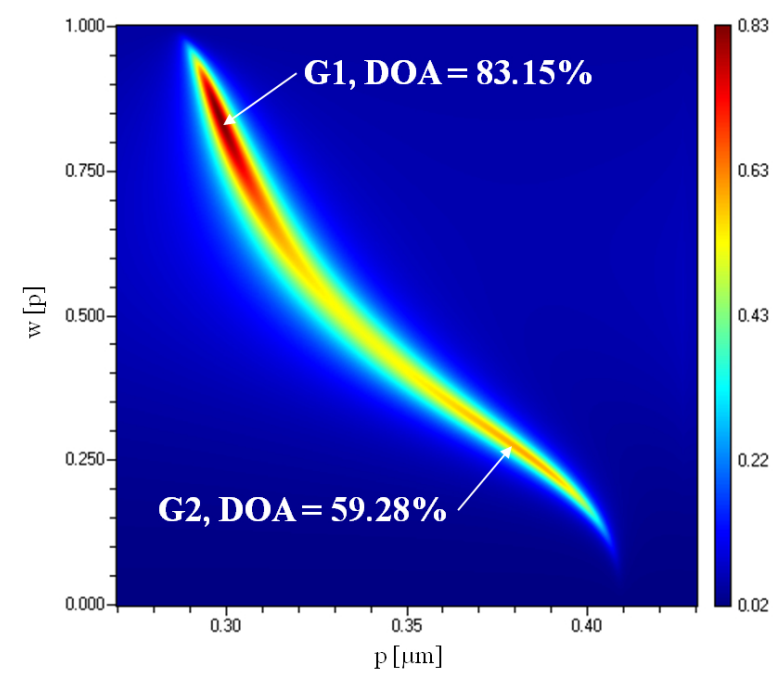

FIG. 8 Parameter scan of global absorption at normal incidence of TM-polarised light (wavelength $\lambda=850 \mathrm{~nm}$ ). The global absorption values are normalised with respect to the incident energy. All Simulations were done with a resolution of 21 modes.

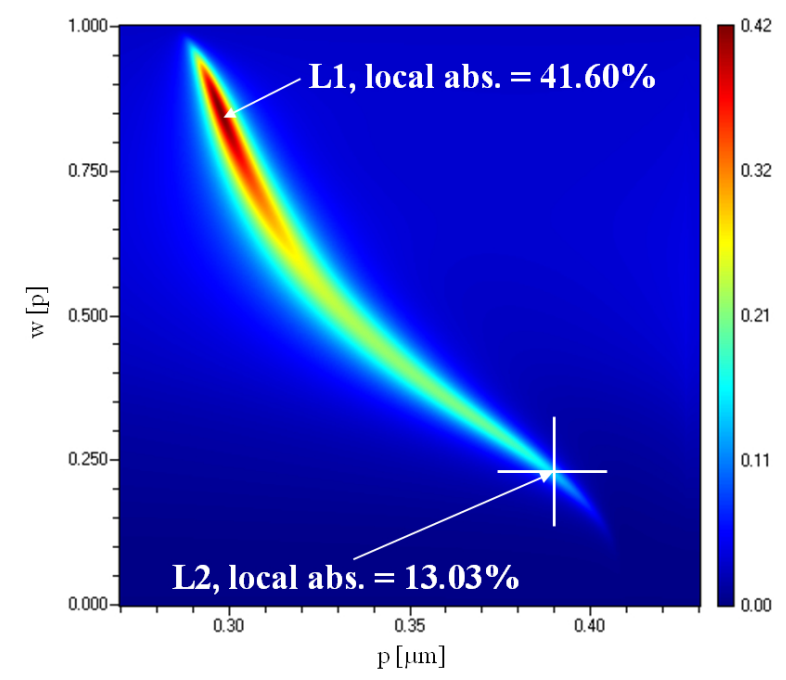

FIG. 9 Local absorption in grating (TM-polarised light).

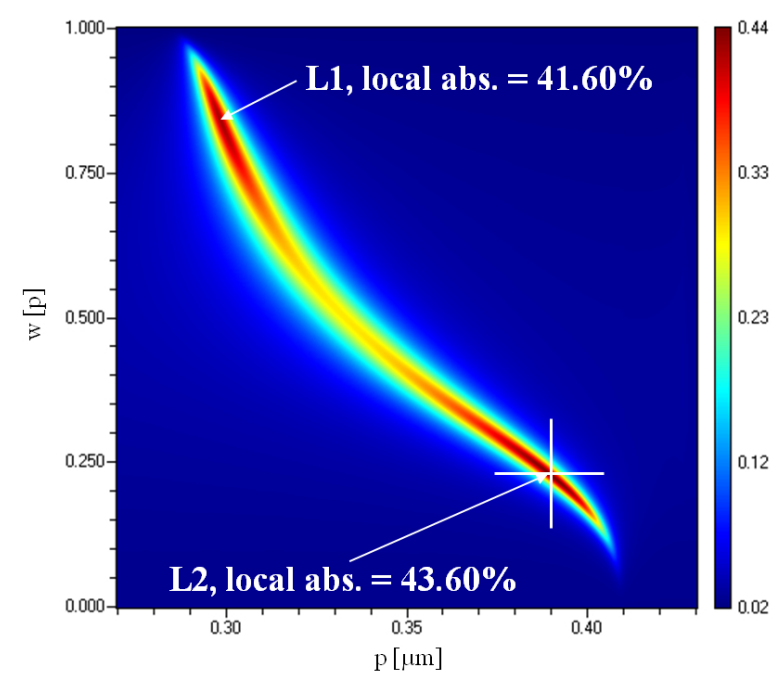

FIG. 10 Local absorption in the active channel (TM-polarised light).

most dissapears in the grating (cf. L2: 13.03\% in Figure 9) and only concentrates in the active channel (cf. L2: 43.6\% in Figure 10). However in the TM-case both L1 and L2 fall short
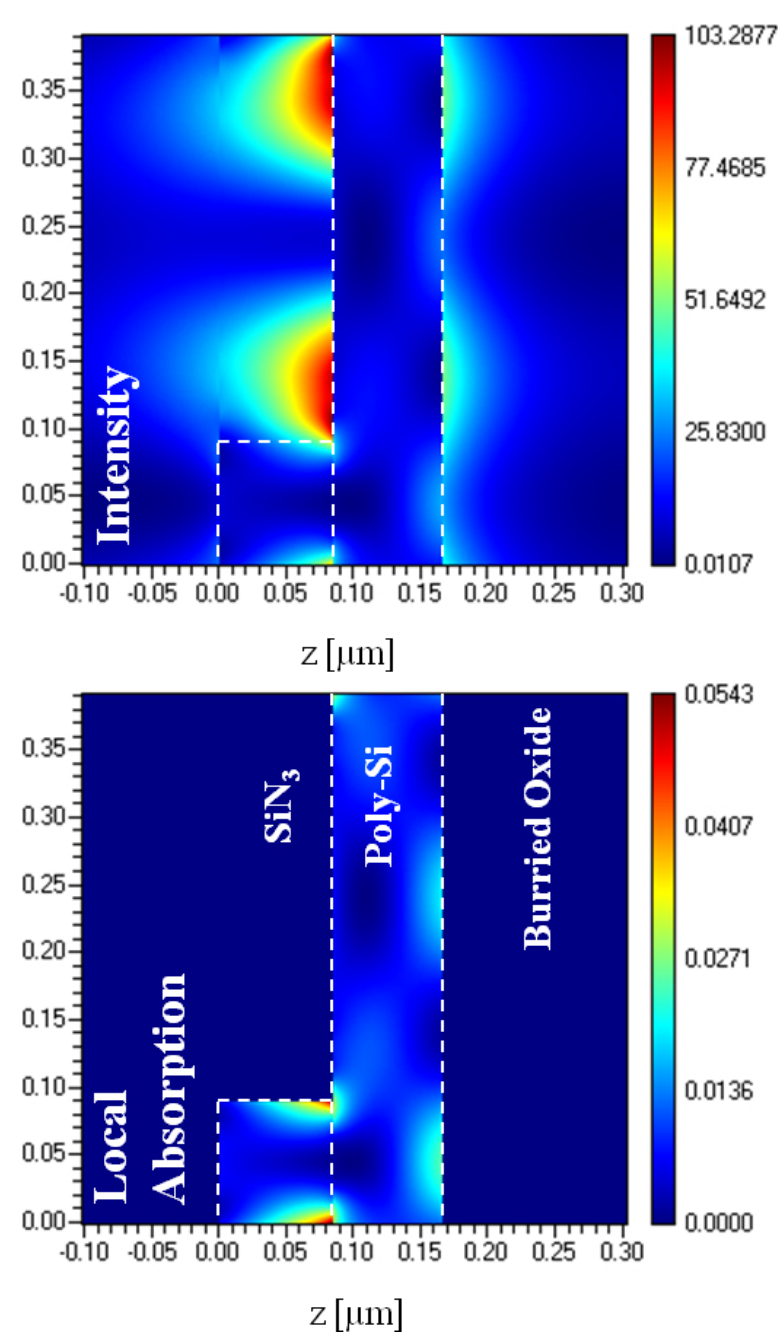

FIG. 11 Intensity and local absorption at $\mathrm{C}_{2}(p=0.39 \mu \mathrm{m}$ and $w=0.23 p)$ (TM-polarised light with normal angle of incidence).

of the TE-case (cf. L1: $68.28 \%$ in Figure 4 and L1: 41.6\%, L2: $13.03 \%$ in Figure 9). Figure 11 illustrates that the intinsity maximum for L1 in TM-polarisation is located in the $\mathrm{SiN}_{3}$-region and outside the structure. Moreover the local absorption does not show the nice symmetric shape and centered position under the gate as in the TE-case.

\section{CONCLUSION}

In this paper we have demonstrated an efficient photodetector concept with enhanced responsivity based on laterally resonant grating structures. The fabrication is fully compatible with the SOI CMOS process. With this approach, we can utilise the existing layers of the standard CMOS fabrication process. Due to the resonant enhancement, sufficient photo current generation can also be achieved in thin layers, resulting in faster photodiodes. By applying the recently devoloped theory of local absorption [1], we were able to optimise the absorption inside the depletion-region of the diodes. The optimisations were done for $850 \mathrm{~nm}$ wavelength and a layer thickness of less than $100 \mathrm{~nm}$. We investigated grating configurations for TE-polarisation as well as for TM-polarisation. Thereby we discoverd that a TM-optimised grating does not qualify for an improved photodetector. In case of an TE-optimised grating though, we have achieved an 
enhancement of efficiency by a factor of 32. Beyond that the resulting grating offers a good local concentration of intensity and absorption which is ideal for a photodetector.

\section{References}

[1] K.-H. Brenner, "Aspects for calculating local absorption with the rigorous coupled-wave method" Opt. Express 18, 10369-10376 (2010).

[2] Ch. Budzinski, R. Güther, and B. Kleemann, "Radiation resistant gratings and their optical properties" Optik 87, 121-125 (1991).

[3] N. Moll, T. Morf, M. Fertig, T. Stöferle, B. Trauter, R. Mahrt, J. Weiss, et. al., "Polarization-Independent Photo-Detectors with Enhanced Responsivity in a Standard SOI CMOS Process" J. Lightwave Technol. 27, 4892-4896 (2009).
[4] Y. M. Song, J. S. Yu, and Y. T. Lee, "Antireflective submicrometer gratings on thin-film silicon solar cells for light-absorption enhancement" Opt. Lett. 35, 276-278 (2010).

[5] J. Heroux, X. Yang, and W. Wang, "GalnNAs resonant-cavityenhanced photodetector operating at $1.3 \mu \mathrm{m}$ " Appl. Phys. Lett. 75, 2716 (1999).

[6] M. S. Ünlü, and S. Strite, "Resonant cavity enhanced photonic devices" J. Appl. Phys. 78, 607 (1995). 